Mar. Drugs 2004, 2, 147-153

Marine Drugs

ISSN 1660-3397

www.mdpi.net/marinedrugs/

\title{
Antimicrobial Activity of Untenospongin B, a Metabolite from the Marine Sponge Hippospongia communis collected from the Atlantic Coast of Morocco
}

\author{
Saida Rifai ${ }^{1}$, Aziz Fassouane ${ }^{1}$, Anake Kijjoa ${ }^{2, *}$ and Rob Van Soest ${ }^{3}$ \\ ${ }^{1}$ Faculté des Sciences, University Chouaib Doukkali, Eljadida, Morocco \\ ${ }^{2}$ ICBAS-Instituto de Ciências Biomédicas de Abel Salazar and CIIMAR, Universidade do Porto, \\ 4099-003 Porto, Portugal. Tel. + 351-22-2062288, Fax + 351222062232 \\ ${ }^{3}$ Institute for Biodiversity and Ecosystem Dynamics, University of Amsterdam, P.O. Box 94766, \\ 1090-GT, Netherlands \\ * Author to whom correspondence should be addressed; Email: ankijjoa@icbas.up.pt
}

Received: 12 July 2004 / Accepted: 28 July 2004 / Published: 25 August 2004

\begin{abstract}
Untenospongin B isolated from the marine sponge Hippospongia communis has been tested for its antimicrobial activity against bacteria and human pathogenic fungi using agar disk method and was found to possess a broad and strong activity toward the test organisms. Its antifungal activity was further characterized by determination of the minimum inhibitory concentration (MIC) against five fungal species using broth microdilution method.
\end{abstract}

Keywords: Porifera, Hippospongia communis, antimicrobial activity, (-)- untenospongin B.

\section{Introduction}

Incidence of fungal infection is emerging worldwide and despite treatment, mortality remains high [1]. Many studies have shown an increasing frequency of systemic infections within the last decades in advanced Human Immunodeficiency Virus infected patients and other patients with deficient immune systems [2]. 
Currently, very few antifungal agents are available and their use may be limited by dangerous side effects [3, 4]. Furthermore, with the emergence of new triazol-resistant strains of fungi, new compounds must still be screened in search of fungicidal effect with a broad spectrum of activity and without side effects.

In the marine environment, sponges (Porifera) are one of the richest sources of both biologically active secondary metabolites and chemical diversity $[5,6]$. These natural products may play a role in warding off predators, and perhaps they also repel fouling organisms. Marine sponges are also a well known source of a unique class of metabolites collectively known as C21 bisfuranoterpenes [7, 8, 9]. Early examples included (+)- furospongin-1 from Mediterranean Spongia officinalis as well as (-)-untenospongin A and B from an Okinawan Hippospongia sp. It is worth noting that (-)untenospongin B is very closely related to another sponge metabolite (+)-tetrahydrofurospongin-1 whose stereochemistry has been well established.

During our continuing studies on bioactive substances from the Moroccan marine organisms, ()-untenospongin B (1) was isolated from the antifungal extract of the marine sponge Hippospongia communis.

Although several biological activities were reported for bisfuranoterpenes there are no studies concerning the antifungal activity of (-)-untenospongin $\mathrm{B}$ against human pathogenic fungi. So the aim of this study was to investigate the antimicrobial activity of (-)-untenospongin B against human pathogenic fungi and bacteria.

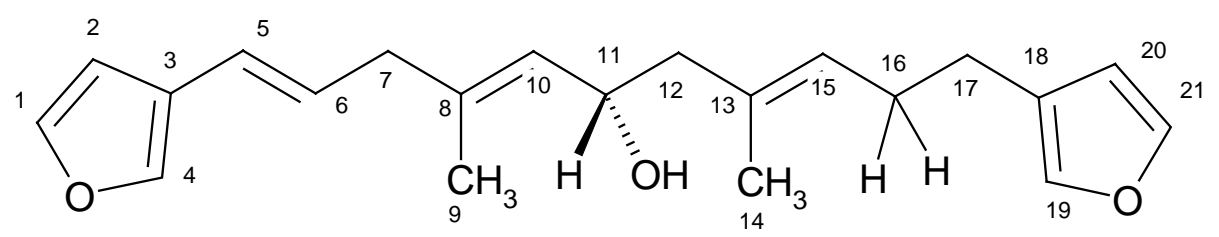

\section{(-)-untenospongin B (1)}

\section{Results and discussion}

The antimicrobial activity of (-)-untenospongin B, isolated from the Moroccan collection of the marine sponge Hippospongia communis, was evaluated against three bacteria, three yeasts and five filamentous fungi at concentration of $100 \mu \mathrm{g} /$ disk. The results of its antibacterial and antifungal activity are summarized in Table 1. (-)-Untenospongin B has shown a broad antimicrobial activity exhibiting antibacterial activity against Escherichia coli, Bacillus subtilis as well as against the ichthyopathogenic strain Vibrio anguillarum. (-)-Untenospongin B showed also an in vitro antifungal activity against Candida albicans, Aspergillus fumigatus, and some other fungi. Light microscopy observations of the zone of fungal growth treated with (-)-untenospongin B showed the presence of non germinated cells and hyphae presenting morphological alterations, such as protoplast ejection and condensation of cytoplasm with empty spaces. 
Table 1. In vitro antibacterial and antifungal activity of (-)-untenospongin B

\begin{tabular}{ccc}
\hline Test organism & \multicolumn{2}{c}{ Growth inhibition diameter (mm) } \\
\cline { 2 - 3 } & $\begin{array}{c}(-)- \\
\text { untenospongin B }\end{array}$ & Amphotericin \\
\hline Bacteria & & \\
Escherichia coli & 13 & ND \\
Bacillus subtilis & 15 & ND \\
Vibrio anguillarum & 9 & ND \\
Yeast & & \\
Candida albicans & 17 & 17 \\
Candida tropicalis & 5 & 0 \\
Cryptoccocus neoformans & 0 & 15 \\
Filamentous fungi & & 20 \\
Aspergillus fumigatus & 17 & 13 \\
Aspergillus niger & 14 & ND \\
Arthoderma simii & 20 & ND \\
Trichophyton rubrum & 13 & \\
& & \\
\hline
\end{tabular}

ND: not determined

Values corresponding to MIC90 (The lowest concentration causing at least $90 \%$ of growth inhibition when compared to drug-free control) of (-)-untenospongin B and amphotericin B are given in Table 2. The results showed that the test medium had a significant influence on the MIC values. The best antifungal activity of (-)-untenospongin B for all species of fungi tested was obtained on YMA medium. Indeed, several studies have previously shown that the composition and the $\mathrm{pH}$ of the test medium as well as other factors such as inoculum size, time, and temperature, constitute the major variables for antifungal susceptibility $[10,11]$.

Comparing the antifungal activity of (-)-untenospongin B with amphotericin B, it was found that (-)-untenospongin B was more active than amphotericin B in inhibiting the growth of C. tropicalis (R2 CIP 1275.81) and F. oxysporum (CIP 108.74). On the other hand, (-)-untenospongin B showed the same activity as amphotericin B in inhibiting the growth of C. albicans (ATCC 10231) and A. niger (CIP 1082.74).

The results of our study were comparable with those of previous publications dealing with the in vitro activity of antifungal agents other than amphotericin B and from chemical compounds currently used in the hospital. For example, the in vitro susceptibility of Fusarium spp. to the new 
triazoles (MIC50 > $8 \mu \mathrm{g} / \mathrm{mL}$ ) determined by Pfaller et al.[10] was less than the in vitro susceptibility of F. oxysporum (CIP 625.72) to (-)-untenospongin B.

Table 2. Minimum Inhibitory Concentration (MIC90) of (-)-untenospongin B and amphotericin $\mathrm{B}$ tested against five fungi.

\begin{tabular}{|c|c|c|c|c|}
\hline \multirow[t]{2}{*}{ Test strain } & $\begin{array}{l}\text { MIC90 of } \\
(\mu \mathrm{g} / \mathrm{ml})\end{array}$ & untenospongin & $\begin{array}{l}\text { BIC90 of } \\
(\mu \mathrm{g} / \mathrm{ml})\end{array}$ & Amphotericin \\
\hline & Casitone & YMA & Casitone & YMA \\
\hline C. albicans & $2-4$ & $0.25-0.5$ & $0.06-0.12$ & $0.5-1$ \\
\hline C. tropicalis & $4-8$ & $2-4$ & $16-32$ & $8-16$ \\
\hline A. fumigatus & $8-16$ & $4-8$ & $8-16$ & $2-4$ \\
\hline A. niger & $8-16$ & $2-4$ & $8-16$ & $2-4$ \\
\hline$F$ & $1-2$ & N.D & $2-4$ & 8-16 \\
\hline oxysporum & & & & \\
\hline
\end{tabular}

\section{Conclusion}

The results obtained from this study suggest that marine natural products isolated from the sponges continue to be an interesting source of antifungal metabolites with better activity and that a strong in vitro antifungal activity of (-)-untenospongin B could be valuable for a development of a new drug for the treatment of fungal infections. Further studies are still needed to determine the toxicity of (-)-untenospongin B and its in vivo activity in the animal model as well as to elucidate the mechanism underlying its activity.

\section{Acknowledgements}

This work was partially supported by the Gabinete de Relações Internacionais da Ciência e do Ensino Superior (GRICES-Portugal) and the Centre National de Recherche Scientifique et Technique (CNRST-Morocco). We thank Prof. Artur Silva of the University of Aveiro for providing the NMR spectra and Mrs. Júlia Bessa of ICBAS for technical assistance.

\section{Experimental}

\section{General}

${ }^{1} \mathrm{H}$ and ${ }^{13} \mathrm{C}$ NMR spectra were recorded at ambient temperature on a Bruker AMC instrument operating at 300.13 and $75.47 \mathrm{MHz}$, respectively. Rotation was determined on a Polax-2 L instrument. EI mass spectra were measured on a Hitachi Perkin - Elmer RMV-6M instrument. 
Silica gel for column chromatography was Si Gel 60 (0.2-0.5 nm Merck), for analytical and preparative TLC Si Gel G-60 254 Merck.

\section{Biological material}

The marine sponge Hippospongia communis was collected in March 2003 at the littoral Atlantic of El-Jadida city, Morocco. The sponge was identified by Dr. Rob van Soest, Institute for Biodiversity and Ecosystem Dynamics, University of Amsterdam. A voucher of the specimen (ZMAPOR17786) was deposited in the collections of the Zoological Museum Amsterdam. The collected material was immediately frozen at $-20^{\circ} \mathrm{C}$ for one night prior to extraction.

\section{Extraction and isolation of (-)-untenospongin B}

The sample (500 g wet weight) was thawed, homogenized with acetone (500 mL), allowed to stand in a dark chamber or 24 hours and filtered. The residue on the filter paper was again extracted with acetone $(3 \times 500 \mathrm{~mL})$. The aqueous acetone extracts were combined, evaporated at reduced pressure until the final volume of the aqueous solution was $c a .100 \mathrm{~mL}$ and then extracted with $\mathrm{CHCl}_{3}\left(3 \times 100 \mathrm{~mL}\right.$ ). The $\mathrm{CHCl}_{3}$ extracts were combined and concentrated at reduced pressure to give a viscous crude $\mathrm{CHCl}_{3}$ extract which was applied to a silica gel 60 column (64 g) and eluted with petrol- $\mathrm{CHCl}_{3}$ and $\mathrm{CHCl}_{3}$-acetone, $250 \mathrm{~mL}$ frs being collected as follows: frs 1-26 ( petrol$\mathrm{CHCl}_{3}, 1: 1$ ), 27-80 (petrol-CHCl 3 , 3:7), 81-97 (petrol- $\left.\mathrm{CHCl}_{3}, 1: 9\right)$, 98-130 $\left(\mathrm{CHCl}_{3}\right.$-acetone,9:1). The ratios refer to $\mathrm{v} / \mathrm{v}$.

Frs 2 and 3 (2.5 g) were combined, applied over Silica gel 60 (15g) and eluted with $\mathrm{CHCl}_{3^{-}}$ petrol, $250 \mathrm{~mL}$ subfrs being collected as follows: subfrs 1-20 (petrol-CHCl 3 , 9:1) and subfrs 21-45 (petrol- $\mathrm{CHCl}_{3}, 4: 1$ ). Purification of subfrs $4-12$ by TLC (Si gel, $\mathrm{CHCl}_{3}$-petrol, 1:1) gave $62 \mathrm{mg}$ of ()-untenospongin $\mathrm{B}$ which was identified by HRMS, ${ }^{1} \mathrm{H}$ and ${ }^{13} \mathrm{C}$ NMR measurements $\left({ }^{1} \mathrm{H},{ }^{13} \mathrm{C}\right.$, DEPTs, COSY, HSQC, HMBC and NOESY) as well as comparison of its proton and carbon chemical shift values and specific rotation with those reported in the literature [12].

(-)-Untenospongin B: Gum; EI-MS, m/z $326 \quad(\mathrm{M}+),. \quad[\alpha]_{\mathrm{D}}^{23}-30.9^{\circ} \quad\left(\mathrm{CHCl}_{3}, \quad \mathrm{c}=\right.$ 0.13g/100ml). ${ }^{1} \mathrm{HNMR}\left(300 \mathrm{MHz}, \mathrm{CDCl}_{3}\right) \delta 7.36 \mathrm{~s}(\mathrm{H}-4), 7.34$ brs (H-1, H-21), 7.21 brs (H-19), 6.50 brs (H-2), 6.27 brs (H-20), $6.25 d(J=15.0, \mathrm{H}-5), 5.89 d d d(J=15.0,7.1,7.1, \mathrm{H}-6), 5.21-5.29 \mathrm{~m}$ (H-10, H-15), 4.44 ddd (J=7.4, 7.4, 7.4, H-11), $2.82 d(\mathrm{~J}=8.2, \mathrm{H}-7), 2.39-2.50 \mathrm{~m}(\mathrm{H}-17), 2.21-2.32$ $m$ (H-16), 1.69s (Me-9), $1.64 \mathrm{~s}(\mathrm{Me}-4) .{ }^{13} \mathrm{CNMR}\left(75.47 \mathrm{MHz}, \mathrm{CDCl}_{3}\right) \delta 143.30(\mathrm{C}-1), 142.72$ (C21), 139.60 (C-4), 138.82 (C-19), 136.91 (c-8), 132.18 (C-13), 128.07 (C-10), 128.06 (C-15), 127.54 (C-6), 124.58 (C-18), 124.20 (C-3), 121.09 (C-5), 110.86 (C-20), 107.48 (C-2), 65.71 (C-11), 48.04 (C-12), 42.83 (C-7), 28.40 (C-16), 24.73 (C-17), 16.68 (C-9), 16.17 (C-14).

\section{Biological activity}


Three bacteria and eight fungal species obtained from the collection of microorganisms of the Pasteur Institute (CIP) and from the American Type Culture Collection (ATCC) were used as the antimicrobial test strains: Bacillus subtilis (ATCC 66331), Escherichia coli (CIP 54127), Vibrio anguillarum (ATCC 19264), Candida albicans (ATCC 10231), Candida tropicalis (R2 CIP 1275.81, an amphotericin B and nystatin resistant strain), Cryptococcus neoformans (ATCC 11576), Aspergillus fumigatus (CIP 1082.74), Aspergillus niger (ATCC 16404), Fusarium oxysporum (CIP 625.72), Trichophyton rubrum (CIP 2043.92) and Arthroderma simii (CIP 902). The bacterial strains were maintained on the LB agar medium, the yeasts on the Sabouraud's agar medium and the filamentous fungi were maintained on malt agar medium at $28^{\circ} \mathrm{C}$. The agar disk diffusion method according to Lemriss et al. [13] was used to test antimicrobial activity of (-)-untenospongin B using casitone agar medium.

The bacteria and yeast inoculum was prepared by suspending in $9 \mathrm{~mL}$ of sterile water for colonies from 24 hours culture on LB and Sabouraud's agar medium respectively. For the filamentous fungi, the inoculum was prepared with the spores derived from 5 to 15 days culture on malt agar medium. The mycelia were covered with $10 \mathrm{~mL}$ of distilled water and the conidia were scraped using sterile pipette. The spores were recovered after filtration on sterile absorbent cotton and were resuspended in sterile distilled water.

The cell density of each inoculum was adjusted with hemocytometer in order to obtain a final concentration of approximately $10^{4} \mathrm{UFC} / \mathrm{mL}, 10^{5} \mathrm{UFC} / \mathrm{mL}$ and $10^{6} \mathrm{UFC} / \mathrm{mL}$ for the yeast, the filamentous fungi and for the bacteria, respectively.

Amphotericin B (Sigma) was used as positive control for yeast and fungi. Each disk contained $100 \mu \mathrm{g}$ of (-)-untenospongin B or amphotericin B was applied on casitone agar media which were previously inoculated with each test strain. Plates were first kept at $4{ }^{\circ} \mathrm{C}$ for at least 2 hours to allow the diffusion of chemicals, and then incubated at $28^{\circ} \mathrm{C}$. Inhibition zones were measured after 24 hours of incubation for bacteria and C. albicans and after 48 hours of incubation for the other fungi.

The microdilution was used to evaluate the minimum inhibitory concentration (MIC) of (-)untenospongin B. The YMA liquid medium and casitone liquid medium were used as test media. Tests were performed in 96-well round bottom sterile culture plates. The suspensions of yeast and filamentous fungi were adjusted in sterile water to match the density of a 0.5 McFarland Standard. The wells of a microdilution plate were inoculated with $180 \mathrm{~mL}$ of the culture medium containing a final inoculum of 0.5-2.5 $10^{3} \mathrm{CFU} / \mathrm{mL}$. (-)-Untenospongin B and amphotericin B previously solubilized in DMSO were serially diluted two folds in the liquid medium to give a range of concentration from 640 to $0.1 \mu \mathrm{g} / \mathrm{mL}$. Twenty $\mu$ l of each concentration were added to wells containing culture suspension except the growth control well. The final concentration ranged from 64 to $0.01 \mu \mathrm{g} / \mathrm{mL}$. Plates were incubated at $35^{\circ} \mathrm{C}$ for 48 hours. Fungal growth was assessed at 494 $\mathrm{nm}$ by measuring the optical density in each well using an enzyme immunoassay multiwell reader (Sigma diagnostic). 


\section{References}

1. Dannaoui, E.; Lortholary, O.; Dromer, F. Technique des associations d'antifongiques in vitro et in vivo chez l'animal. J. Mycol. Med. 2003, 13, 73-85.

2. Thiebaut, A. Empirical treatment by antifungal drugs in febrile neutropenia. J. Mycol. Med. 2002, 12, 115-119.

3. Lorthoraly, O.; Denning, W. D.; Dupont, B. Endemic mycoses: a treatment update. J. Antimicrob. Chemother. 1999, 43, 321-331.

4. Andriole, V.T. Current and future antifungal therapy: new targets for antifungal agents. $J$. Antimicrob. Chemother. 1999, 44, 151-162.

5. Kijjoa, A.; Sawangwong, P. Drugs and cosmetics from the Sea. Mar. Drugs 2004, 2, 73-82.

6. Proksch, P.; Ebel, R.E.; Ebel, R. Drugs from the sea-opportunities and obstacles. Mar. Drugs 2003, 1, 5-17.

7. Fontana, A.; Albarella, L.; Scognamiglio, G.; Uriz, M.; Cimino, G. Structural and stereochemical studies of C-21 terpenoids from Mediterranean Spongiidae sponges. J. Nat. Prod. 1996, 59, 869-872.

8. Rochfort, S. J.; Atkin, D.; Hobbs, L.; Capon, R.J. Hippospongins A-C : new furanoterpenes from Australian marine sponge Hippospongia sp. J. Nat. Prod. 1996, 59, 1024-1028.

9. Capon, R. J.; Jekins, A.; Rooney, F.; Ghisalberti, E. L. Structure revision and assignment of absolute stereochemistry of a marine C21 bisfuranoterpene. J. Nat. Prod. 2001, 64, 638-639.

10. Ryder, N.S.; Wagner, S.; Leitner, I. In vitro activities of terbinafine against cutaneous isolates of Candida albicans and others pathogenic yeasts. Antimicrob. Agents Chemother 1998, 42, 1057-1061.

11. Pfaller, M.A.; Messer, S.A.; Boyken, L.; Huynh, H.; Hollis, R.J.; Diekema, D.J. In vitro activities of 5-fluorocytosine against 8803 clinical isolates of Candida spp.: global assessment of primary resistance using national committee for clinical laboratory standards susceptibility testing methods. Antimicrob. Agents Chemother 2002, 46, 3518-3521.

12. Umeyama, A.; Shoji, N.; Arihara, S.; Ohizumi, Y.; Kobayashi, J. Untenospongin A and B, Novel furanoterpenes with coronary vasodilating activity from the Okinawan marine sponge Hippospongia sp. Aust. J. Chem. 1989, 42, 459-462.

13. Lemriss, S.; Marquet, B.; Ginestet, H.; Lefeuvre, L.; Fassouane, A.; Boiron, P. Screening of new antifungal compounds in a collection of chemical products. J. Mycol. Med. 2003, 13, 189-192.

Sample Availability: Samples are available from the authors.

(c) 2004 by MDPI (http://www.mdpi.org). Reproduction is permitted for noncommercial purposes. 